\title{
6. COMMISSION DES TELEGRAMMES ASTRONOMIQUES
}

\author{
Président: M. A. A. Mikhailov. \\ Directrice DU BUREAU: Mlle Vinter Hansen. \\ Membres: MM. Cunningham, Hogg, Spencer Jones, Platzeck, van den Bos, Director \\ Harvard Observatory.
}

The members of Commission 6 have made no suggestions for a modification or improvement of Astronomical Telegrams and Announcement Cards. According to a proposal by Sir Harold Spencer Jones the preliminary co-ordinates of the Earth's pole derived at the Central Bureau of the International Latitude Service are now published in the Circulars. These may be used in the Time Service and for the determination of geographic positions.

\section{A. Mrkhailov \\ President of the Commission}

\section{Report of the Central Bureau for Astronomical Telegrams}

The work of the Bureau since I952 has proceeded along the usual lines. As the number of subscribers to telegrams and circulars, and to circulars only, is steadily growing, the clerical work at the Bureau is now quite considerable, and it would not have been possible to keep the present price of subscription fairly low, if we were not the recipients of subsidies from the International Astronomical Union and the Danish Rask-Orsted Foundation, which subsidies pay for all our clerical work. Some astronomers, notably Dr L. E. Cunningham, Dr G. Merton with the computers of the B.A.A., Dr L. Kresák and some Japanese computers give the Bureau great help in supplying orbits and ephemerides of current interest.

The Bureau tries its best to satisfy the desires of the subscribers. A few of the telegram subscribers wish to have all news transmitted by telegram, while most of the subscribers only wish first positions of comets and first notices of novae conveyed telegraphically. Also quite a few make restrictions regarding the magnitude of the object, for which they want news sent by telegram. Further, some overseas subscribers to the circulars want the circulars sent by air-mail, for which service they pay the extra costs.

While formerly all subscribers ordered the telegrams and circulars direct from the Bureau, and all subscriptions were considered continuous until specifically cancelled, some subscribers now order the circulars through an authorized book agency in their own country and only for a year at a time. I should like to point out that as a rule such arrangement is not to the advantage of the subscribers, besides giving the Bureau some extra clerical work, for the said book agencies cannot be expected to understand the workings of the service given by the Bureau and often do not renew the subscriptions in due time. Hence gaps may occur in the mailing of circulars to the subscribers involved, and these often complain about the non-appearance of the circulars at the beginning of a new year, so much correspondence is needed between the Bureau on the one hand and the subscriber and book agency on the other. The Bureau would therefore appreciate it if the subscribers would deal direct with the Bureau as much as possible.

Below are given the number of telegrams received and transmitted to the subscribers in the period r952-54 (20 October), together with the number of circulars issued in the same period. Further, a list is given of present subscribers to the services of the Bureau:

\begin{tabular}{|c|c|c|c|}
\hline & \multicolumn{2}{|c|}{ Telegrams } & \multirow[b]{2}{*}{ Circulars } \\
\hline & Received & Sent & \\
\hline 1952 & 35 & 571 & $47(1340-1386)$ \\
\hline 1953 & 27 & 319 & 48 (1387-1434) \\
\hline 1954 (until 20 Oct.) & 34 & 525 & $44(1435-1478)$ \\
\hline & $\overline{96}$ & $\overline{1415}$ & $\overline{139}$ \\
\hline
\end{tabular}




\section{Subscribers to Telegrams and Circulars}

Harvard College Observatory, Cambridge, Massachusetts, U.S.A.

Union Observatory, Johannesburg, South Africa.

Commonwealth Observatory, Mount Stromlo, Canberra, Australia.

Astronomitcheskij Institut im. Sternberga, Nowo-Waganjkowskij per 5, Moskva 22, U.S.S.R.

Astronom. Rechen-Institut, Grabenstrasse 14, 17 a-Heidelberg, Germany.

Royal Observatory, Edinburgh 9, Scotland.

Royal Greenwich Observatory, Hailsham, Sussex, England.

Norman Lockyer Observatory, Salcombe Regis, Sidmouth, S. Devon, England.

Observatorio Fabra, Calle Aragon $2192^{\circ} 2$ A, Barcelona, Spain.

Observatorio astronomico, Alfonso XII 5, Madrid, Spain.

Observatorio de Marina, San Fernando, Spain.

Observatoire de l'Université de Bordeaux à Floirac, Gironde, France.

Observatoire, Place Leverrier, Marseille, France.

Observatoire National, Paris I4, France.

Observatoire, I I Rue de l'Université, Strasbourg, Bas-Rhin, France.

Observatoire d'Alger, Bouzaréa, Alger, Africa.

Sterrewacht, Leiden, Holland.

Osservatorio Astronomico Capodimonte, Napoli, Italy.

Osservatorio Astronomico di Brera, Milano, Italy.

Osservatorio Astronomico delle Università, Padova, Italy.

Observatoire, Bucarest, Roumania.

Observatoriet, Stockholm-Saltsjöbaden, Sweden.

Observatoire, Genève, Switzerland.

Observatory, Helwan, Egypt.

Observatoire Royal, Uccle, Belgium.

Observatoire astronomique de Tokyo, Mitaka près Tokyo, Japan.

Observatoire d'Astronomie physique, Meudon, S. et O., France.

Royal Astronomical Society, Burlington House, Piccadilly, London, W. I, England.

Osservatorio astronomico, Monte Mario, Roma, Italy.

Observatory, Athens, Greece.

Osservatorio astronomico, Via G.P. Tiepolo 3, Trieste.

Obserwatorium Uniwersitetu Poznań, Ul. Sloneczna 36, Poznań 8, Poland.

Observatoriet, Uppsala, Sweden.

Observatoire Astronomique, Veliki Vračar, Beograd, Yougoslavia.

Specola Vaticana, Castel Gandolfo, Roma, Italy.

Observatorio astronomico da Universidade de Coimbra, Portugal.

Observatoriet, Lund, Sweden.

Observatoriet, Yliopisto, Turku, Finland.

Observatorio Nacional, Morro Sao Januario, Rio de Janeiro, Brazil.

Institut Astronomique, Sofia, Bulgaria.

Ole Römer-Observatoriet, Aarhus, Denmark.

Institut d'Astrophysique, 98 bis Boulevard Arago, Paris I4, France.

Observatoire de Haute Provence, Saint-Michel l'Observatoire, Basses-Alpes, France.

Dr Leland Cunningham, Leuschner Observatory, Berkeley 4, California, U.S.A.

Astronomisches Institut, Muesmattstrasse 25, Bern, Switzerland.

The University of London Observatory, Mill Hill Park, London, N.W. 7, England.

Osservatorio astronomico della Università, Bologna, Italy.

B.A.A., Oxford, England.

Dr E. Leutenegger, Rüeggerholzstraisse I7, Frauenfeld, Switzerland.

The Observatories, Cambridge, England.

Dunsink Observatory, Co. Dublin, Eire.

Carter Observatory, Wellington, W. I, New Zealand.

Armagh Observatory, Armagh, Northern Ireland. 
Konkoly Observatory, Konkoly Thege Miklós-Út 13-17, Budapest X1I, Hungary.

Observatoire de la Société Astronomique de France, 28 Rue Serpente, Paris 6e, France.

Universitäts-Sternwarte, Türkenschanzstrasse I7, Wien I Io, Austria.

Institut d'Astronomie de l'Université de Lausanne, Cité-devant 2, Lausanne, Switzerland.

C.S.I.R.O. (Radiophysics), University Grounds, City Road, Chippendale, N.S.W., Australia.

Dr D. J. Febrer, Paseo Bonanova I I, Barcelona, Spain.

Slovenská akadémia vied Astronomický ústav na Skalnatom Plese, Czechoslovakia.

Kniznica Slovenskej akad. vied, Klemensova 27, Bratislava, Czechoslovakia.

Laboratoire d'Astronomie de la Faculté des Sciences de Lille, r Rue du Fg de Douai, Lille,

France.

Astronomický ústav CSAV, Ondřejov, Czechoslovakia.

Astronomický ústav, Budečská 6, Praha I2, Czechoslovakia.

\section{Subscribers to Circulars}

Instituttet for teoretisk Astrofysikk, Blindern, V. Aker, Norway.

Observatoire astronomique de l'Université de Jassy, Jassy, Roumania.

Lick Observatory, Mount Hamilton, California, U.S.A.

Yerkes Observatory, Williams Bay, Wisconsin, U.S.A.

Mount Wilson Observatory, Pasadena, California, U.S.A.

U.S. Naval Observatory, Washington, D.C., U.S.A.

Serials Department, The General Library, University of California, Berkeley 4 , California, U.S.A.

Observatoire Astronomique, Kopernika 27, Krakow, Poland.

Observatoire, Lyon, St. Genis Laval, France.

Yamamoto Observatory, Ksatsu, Sikagen, Japan.

Brown University Library, Ladd Observatory, 2 ro Doyle Avenue, Providence 6, R.I., U.S.A.

Observatoire de Juvisy, Seine et Oise, France.

Astronomisches Institut der Universität, ul. Dlugosza 8, Lwów, U.S.S.R.

Taschkent Astronom. Observatorium, Komitet Nauk Pri S.N.K., U.S.S.R. Fundamentaln. Biblioteka, ul. Abduly Tukajeva I, g. Taschkent, U.S.S.R.

Sterrewacht, Lembang, Java.

Observatory of the University of Michigan, Ann Arbor, Michigan, U.S.A.

Professor J. F. Cox, Laboratoire d'Astronomie de l'Université libre de Bruxelles, 50 Avenue des Nations, Bruxelles, Belgium.

Observatoire de Toulouse, Toulouse, Haute-Garonne, France.

Institut d'Astrophysique de l'Université de Liège, Cointe-Sclessin, Belgium.

Professor Oswald Thomas, Salesianergasse 8, Wien III, Austria.

H.M. Nautical Almanac Office, Royal Greenwich Observatory, Herstmonceux Castle, Hailsham, Sussex, England.

Hans Q. Rasmusen, Værslevgaarden, Værslev, Denmark.

G. F. Kellaway, Coker Hill, West Coker, Yeovil, Somerset, England.

Nizamiah Observatory, Begumpet, Hyderabad-Deccan, India.

Glavnaja Astronom. Observatorija S.S.S.R. c. Pulkove(G.A.O.), bliz Leningrad, V.O., U.S.S.R.

The Astronomical Society of Edinburgh (Norman G. Matthew), I26 West Savile Terrace, Edinburgh 9, Scotland.

Royal Observatory, Capetown, South Africa.

Astrophysikalisches Observatorium, Potsdam, Germany.

Hamburger Sternwarte, Bergedorf, Germany.

Universitäts-Sternwarte, Potsdam-Babelsberg 2, Germany.

Sternwarte Sonneberg in Thüringen, Germany.

Kwasan Observatory, Yamashina, Kyoto, Japan.

Observatoire, Besançon, France.

J. Peridier, Observatoire Le Houga, Les Arousettes (Gers), France.

Observatorio Astronomico, Cordoba, Argentina. 
Warner and Swasey Observatory, Case Institute of Technology, Taylor and Brunswick Roads, East Cleveland I2, Ohio, U.S.A.

Biblioteka Akademii Nauk, Birgevaya linija I, Leningrad, U.S.S.R.

Dr C. Luplau Janssen, Urania-Observatoriet, Dronning Olgasvej 25, Copenhagen F, Denmark. Astronomiska Observatoriet, Helsingfors, Finland.

Eidgenössische Sternwarte, Zürich, Switzerland.

McDonald Observatory, Fort Davis, Texas, U.S.A.

University of California at Los Angeles, Library, Los Angeles 24, California, U.S.A.

Lowell Observatory, Flagstaff, Arizona, U.S.A.

Astronomical Observatory of Wroclaw University, Kopernika II, Wroclaw, Poland.

Robert A. Naef, 'Orion', Auf der Platte, Meilen (Zürich), Switzerland.

Institut d'Astronomie Pratique de l'Ecole Polytechnique de Varsovie, rue Lwowska 7 , Warszawa, Poland.

Jodrell Bank Experimental Station, Lower Withington, nr. Crewe, England.

Cincinnati Observatory, Cincinnati 8, Ohio, U.S.A.

Sterrewacht 'Sonnenborgh', Zonnenburg 2, Utrecht, Holland.

Professor Dr A. Kaufmann, Sternwarte, Greiben 5, Solothurn, Switzerland.

Instituto de Astronomia, c/o Observatorio Astronomico, Casilla de Correo No. 867, Montevideo, Uruguay.

The Institute for Theoretical Astronomy (Academy of Sciences of the U.S.S.R.), Leningrad, U.S.S.R.

Observatoire du Pic du Midi, Bagnères de Bigorre, Hautes Pyrénées, France.

Universitäts-Sternwarte, Jena, Germany.

Yale University Observatory, Prospect and Canner Streets, New Haven I I, Conn., U.S.A.

Osservatorio astronomico di Torino, Pino Torinese, Italy.

A. Schmuhl, Chr. II's Plads 9, Copenhagen, Denmark.

R. Rigollet, 67 Avenue de Versailles, Paris I6, France.

Biblioteka Akademii Nauk, U. Dzerzhinkogo 8, Tbilisi, U.S.S.R.

Bibliothèque du Bureau des Longitudes, Palais de l'Institut, 3 Rue Mazarine, Paris 6, France.

Universitäts-Sternwarte, Universitäts-Str. 4, Innsbruck, Austria.

California Institute of Technology, Astrophysics Library-Palomar, 120r East California St., Pasadena 4, California, U.S.A.

Department of Astronomy, The University, Manchester 13, England.

L. Michiels, Observatoire de Bièvre, 22.Rue de la Fleur, Borgerhout-Anvers, Belgium.

J. Storms, Auwersstraat 6I, Berchem-Anvers, Belgium.

Biblioteca Academiei R.P.R., Procurares Publicatiilor, Calea Victoriei I25, Bucarest Roumania (2 subscriptions).

Observatorio astronomico de la Universidad de Chile, Casilla 36-D, Santiago de Chile.

Observatoire Mont Gros, Nice, France.

Filial Biblioteki Akademii Nauk S.S.S.R., Baltijsky Poselok 42 b, Moskva 57, U.S.S.R. (2 subscriptions).

University of Pennsylvania Library, 34th Street and Woodland Ave., Philadelphia 4, Pa., U.S.A.

Drzavno Preduzece, Jugoslovenska Knijga, Terazije 27/II, Beograd, Jougoslavia.

Kultura, P.O.B. I, Budapest 72, Hungary.

Moskovskoje Otdelenije Biblioteki Akad. Nauk, 2 Donskoj proezd, Moskva 7r, U.S.S.R. (2 subscriptions).

Gos. Nauk. Biblioteka, Minist. Kultury S.S.S.R., Pl. Nogina 2/5, Moskva, U.S.S.R.

Astronomisch-Meteorologische Anstalt der Universität Basel, Binningen, Switzerland.

Amherst College, Department of Astronomy, Amherst, Massachusetts, U.S.A.

Guozi Shudian, P.O. Box 5o, Peking, China.

Perkins Observatory, Delaware, Ohio, U.S.A.

Observatorio Cagigal, Apartado I 855, Caracas, Venezuela.

Ústav astronomický prírodověd. fak. Masarykovy university, Kotlár̆ská 2, Brno, Czechoslovakia.

Osservatorio Astrofisico di Catania, P. za Vaccarini I I, Catania, Italy. 
P.P.K. 'Ruch', U1. Kielecka 43, Warszawa, Poland (2 subscriptions).

Děkanát fakulty zeměměřičské, Husova 5, Praha I, Czechoslovakia.

Oblastní hvězdárna, Plzeň-Slovany, Czechoslovakia.

Biblioteka Akademii Nauk SSSR, Birgevaia linia I, Leningrad I64, U.S.S.R.

Jugoslovenska Knijga, Terazije 27/I I, Beograd, Yugoslavia.

Ceské vysoké učeni tecknické, ústav astron. a základu geofysiky, Karlovo nám. 13, Praha II, Czechoslovakia.

\section{Report of meeting. 3I August 1955}

Acting President: Miss J. M. Vinter Hansen.

SECRETARY: Dr G. Merton.

The President reported that the accounts of the Bureau had been audited and passed by Sir Harold Spencer Jones.

Dr van den Bos proposed, and Miss F. W. Wright seconded, and the meeting approved of the following resolution:

That the I.A.U. be asked to continue the subvention of 1200 gold francs a year to the Central Bureau for Astronomical Telegrams.

Dr Menzel reported that he had made inquiries among subscribers to the Harvard Announcement Cards as to the working of the Telegraphic Service and it was unanimously agreed that this was satisfactory, and no improvements were suggested.

The President referred to the resolution by Commission I5 asking for a Telegraphic Service giving information on physical details and changes in comets.

Dr Waterfield explained his views and suggestions, and some discussion took place.

It was agreed to accept the request by Commission ${ }_{5}$ that an additional Telegraphic Service be arranged to provide physical information on comets for those who require it. 